\title{
Post extraction inversion slice imaging for 3D velocity map imaging experiments
}

\author{
Felix Allum ${ }^{\mathrm{a}}$, Robert Mason ${ }^{\mathrm{a}}$, Michael Burt ${ }^{\mathrm{a}}$, Craig S. Slater ${ }^{\mathrm{a}}$, Eleanor Squires ${ }^{\mathrm{a}}$, \\ Benjamin Winter ${ }^{\mathrm{a}}$, Mark Brouard ${ }^{\mathrm{a}}$ \\ ${ }^{a}$ The Chemistry Research Laboratory, Department of Chemistry, University of Oxford, 12 \\ Mansfield Road, Oxford OX1 3TA, United Kingdom
}

\author{
ARTICLE HISTORY \\ Compiled October 21, 2020
}

\begin{abstract}
An experimental configuration for velocity map ion imaging experiments is presented, in which a pulsed voltage defocusses the ion Newton sphere along the timeof-flight axis. This significantly spreads the times-of-flight for ions with the same mass-to-charge ratio, allowing for either sliced or three-dimensional velocity imaging with high slicing resolution along the time-of-flight axis. The technique is coupled to an event-triggered, position-sensitive sensor, enabling full three-dimensional Newton sphere imaging at high count rates, with significantly improved slicing resolution $(\sim 1-2 \%)$ compared to previous DC slicing approaches. Furthermore, good slice imaging conditions can be brought about at relatively high extraction voltages, circumventing issues regarding image size, the effect of stray fields, and poor detection efficiency when operating at low extraction voltages. The method, termed Post Extraction Inversion Slice Imaging (PEISI) was designed and optimized through ion trajectory simulations and experimentally verified on the well-studied photodissociation of OCS at around $230 \mathrm{~nm}$. We demonstrate that this approach is suitable for recording full 3D angular distributions of systems lacking an axis of cylindrical symmetry in the detector plane, for which conventional image inversion techniques are not valid. We believe this method could be useful in a range of studies of systems lacking cylindrical symmetry, including studies into angular momentum polarization and bimolecular scattering.
\end{abstract}

\section{KEYWORDS}

Velocity map imaging, Photodissociation, Ion imaging, Ion optics, Post-extraction differential acceleration

\section{Introduction}

The techniques of ion-imaging and the closely related velocity map ion imaging (VMI), first introduced by Chandler and Houston [1] and Eppink and Parker [2], respectively, are widely applicable tools in the study of gas-phase molecular reaction dynamics. The imaged nascent product velocity distributions following a photodissociation or scattering process can be directly related to features of the potential energy surface(s) over which the system evolves. Consequently, VMI has been applied to great effect in detailed studies into the dynamics of molecular photodissociation and scattering processes $[3,4]$. 
In the original work of Eppink and Parker, an array of three open electrodes (termed the repeller, extractor, and ground electrodes) act as an ion lens. With suitable voltages applied to the repeller and extractor, the transverse velocity of ions are mapped onto a position-sensitive detector. The resulting images are therefore a two-dimensional projection of a three-dimensional velocity distribution, as a result of flattening the ion Newton sphere in the detector plane. If the velocity distribution possesses cylindrical symmetry, it is possible to reconstruct the 3D distribution through a number of methods, generally based on the inverse Abel transform [5-9]. However, these procedures add noise to images, and the condition of cylindrical symmetry often is not met experimentally, for instance in studies of angular momentum polarization [10-12] or bimolecular scattering $[13,14]$. As a result, efforts have been made to circumvent this process by directly imaging the central slice of a Newton sphere [15-18].

Several experimental approaches to 'slice' imaging exist, generally with a common goal of stretching the ion cloud along the time-of-flight axis prior to its arrival at the detector. The detector can then be gated over the arrival time of the central slice of the sphere. A widely applicable technique, DC slicing, was developed independently by Townsend et al. [16] and Lin et al. [17]. In these methods, additional electrode(s) are incorporated into the ion optics assembly to create a lower potential difference in the extraction region, whilst still bringing about velocity mapping conditions. Consequently, there is a greater turnaround time for fragments produced with negative velocities along the time-of-flight axis, and so the arrival time spread, $\Delta t$ for a given ion increases. Typically, $\Delta t$ is of the order of several hundred nanoseconds, with the gate time of the detector, $\delta t$ on the order of tens of nanoseconds, giving a slicing resolution, $\delta t / \Delta t$ of $\sim 10 \%$. Recently, significant progress has been made in correcting for the effects of finite slicing resolution using the finite slice analysis technique $[8,19]$. In an alternative approach to slice imaging introduced by Kitsopoulos and coworkers [15], a delay between ionization and extraction is introduced by pulsing the (gridded) extraction electrodes from ground. During this delay, the ion cloud expands under field-free conditions, leading to a larger ion sphere impinging on the detector following velocity mapping, with a greater $\Delta t$. The ability to effectively slice image over large interactions using the delayed extraction technique has proven beneficial in studies of scattering at surfaces [20]. In both DC and delayed extraction slice imaging, varying the time window over which ion events are recorded results in imaging sequential slices through the three-dimensional velocity distribution. By incorporating a fast timestamping detector, many such slices can be recorded simultaneously, as demonstrated previously using a Pixel Imaging Mass Spectrometry camera [21-23] or a TimePix detector [24-27]. Similar techniques involving the correlation of events recorded by a camera and a digitized high-resolution time-of-flight trace have similarly realised the goal of three-dimensional imaging at high count rates [27-30].

Slice imaging has proved to be a very powerful experimental technique and has been used in a range of pioneering studies into gas-phase molecular dynamics [3138]. However, there are some experimental limitations of the previously described slice imaging approaches. In the case of DC slice imaging, increasing the arrival time spread for a given ion can only be achieved by lowering the repeller voltage. However, doing so also increases the radius of the ion cloud in the detector plane, and so often the requirement that the cloud does not exceed the size of the detector becomes a limiting factor. Furthermore, very low repeller voltages adversely affect the ion detection efficiency of a MCP/phosphor screen stack [39-41], which depends on the impact velocity of ions. Finally, trajectories of slow ions are more greatly affected by any stray fields within the spectrometer, which can often be difficult to fully eliminate. This is 
also a significant possible issue in the delayed extraction slicing method, in which ions spend time under 'field-free' conditions, where trajectories are most easily perturbed by stray fields. The use of grids for the extraction and repeller electrodes may also lead to trajectory distortions of the order of the grid spacing, a potentially significant issue in the extraction region when the ion cloud is at its smallest. There is also the consideration that, if the slice through the Newton sphere is insufficiently narrow, complications can arise in the quantitative analysis of the sliced image, particularly in the case of detection of polarized photofragments. As discussed in more detail in the next section, the Post Extraction Inversion Slice Imaging (PEISI) method tackles each of these limitations, allowing tuneable Newton sphere stretching along the timeof-flight axis with relatively high extraction fields, open extraction electrodes and the ability to expand the Newton sphere along the time-of-flight axis without transverse stretching. This is easily achieved by modifying a conventional DC slice imaging spectrometer, adding two additional electrodes. The PEISI method and the simulations used to optimize and characterize it are described in the following section, before experimental verification on the well-studied system of the UV photodissociation of OCS [35, 42-55], imaging the $\mathrm{CO}^{+}$and $\mathrm{S}^{+}$ions resulting from photodissociation and subsequent resonance-enhanced multiphoton ionization (REMPI) at around $230 \mathrm{~nm}$. Detailed analysis extracting a photofragment angular distribution directly from the recorded three-dimensional data by expansion in spherical harmonics is then presented, highlighting potential applications of this experimental approach.

\section{Concept and simulations}

To record high resolution sliced and/or three-dimensional ion velocity distributions, a wide spread in ion arrival times is required, where the arrival time of a given ion is dependent on its velocity along the time-of-flight axis. Maximising the spread in ion arrival times is the inverse of a problem frequently tackled in mass spectrometry, in which minimizing ion arrival time spread due to spread in initial ion velocity is desired to increase mass resolution. A number of first order velocity focussing schemes have been developed since the original work in this area by Wiley and McLaren [56]. Aoki et al. [57] introduced Post Extraction Differential Acceleration (PEDA), in order to improve mass resolution in stigmatic imaging mass spectrometry. A pulsed voltage is applied following ion extraction, which accelerates ions at the back of the ion sphere relative to those at the front. With the correct pulsed voltage, this creates a temporal focus in the plane of the detector. In PEISI, a much greater potential difference is used to shift the temporal focus closer to the pulsed plate, so that ions beyond this point are defocussed along the time-of-flight axis. The temporally defocussed ion cloud may then be sliced with very high resolution along the time-of-flight axis, either through use of conventional detector gating or a fast-timestamping camera.

Simulated trajectories of a cloud of $\mathrm{CO}^{+}$ions with $0.3 \mathrm{eV}$ kinetic energy are shown in Figure 1, both in a DC slicing setup and the PEISI approach. In both cases a repeller voltage of $1 \mathrm{kV}$ is used, with the repeller, extractor, and third lens held in a $1: 0.886: 0.775$ voltage ratio. In the PEISI case, a $3 \mathrm{kV}$ pulsed voltage is applied at $2.9 \mu \mathrm{s}$. This corresponds to the ion cloud being positioned between the pulsed plate and the ground grid, as highlighted in yellow. Despite both using the same repeller voltage, a far greater arrival time spread is observed in the PEISI case, as a result of the Newton sphere inverting (at the position indicated by the green dashed line) and defocussing along the time-of-flight axis following application of the pulsed voltage. 


\section{a) DC Slicing}

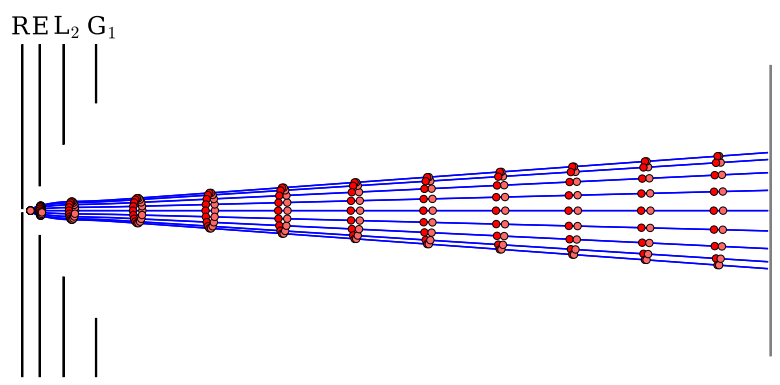

b) PEISI

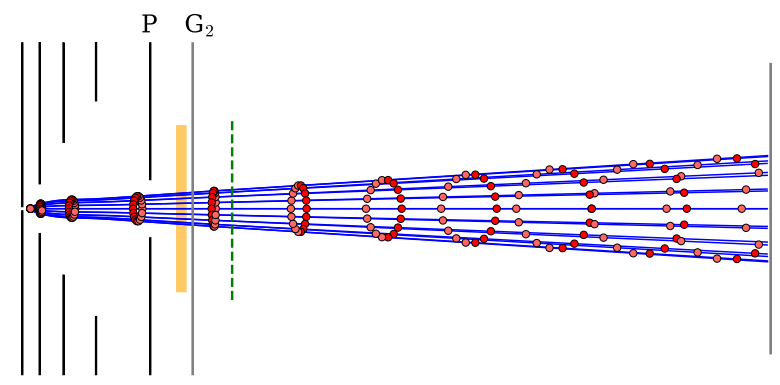

Figure 1. Simulated ion trajectories (blue lines) for a series of $\mathrm{CO}^{+}$ions with $0.3 \mathrm{eV}$ kinetic energy under (a) DC slicing and (b) PEISI conditions. Initial ion velocities are uniformly distributed along the time-of-flight axis. Ion positions at time intervals of $0.85 \mu \mathrm{s}$ are highlighted in dark red for ions with an initially negative velocity along the time-of-flight axis (away from the detector), whereas ions with initially positive velocity along this axis are coloured in pink. In the PEISI case, the spatial extent of the ion cloud upon application of the pulsed voltage is highlighted in yellow, and the plane in which the ion sphere is inverted is shown as a green dashed line.

This elongation of the Newton sphere along the time-of-flight axis is achieved without any additional expansion in the plane of the detector.

a)

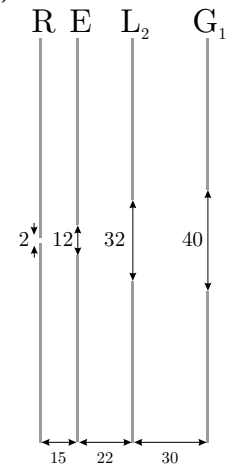

b)

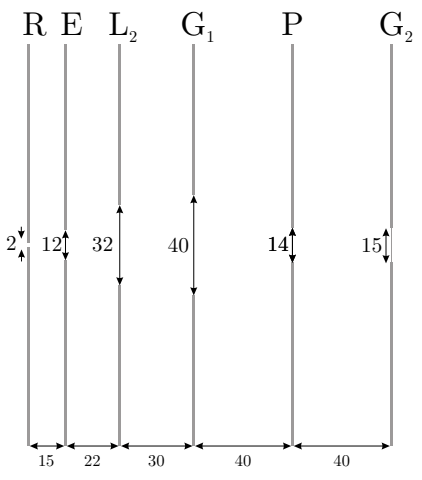

Figure 2. (a) DC slicing and (b) PEISI ion optics geometries. All marked distances are in millimetres, and each plate is $1 \mathrm{~mm}$ thick. R: Repeller, E: Extractor, $\mathrm{L}_{2}$ : Second lens, $\mathrm{G}_{1}$ : Ground plate, $\mathrm{G}_{2}$ : Ground mesh grid, P: Pulsed plate.

In order to determine the optimal experimental parameters for PEISI, ion trajectories were simulated using SIMION 8.1 [58]. Due to the multi-dimensional nature of the optimization a genetic algorithm was employed [59]. The potentials on each electrode, the timing of the pulsed voltage, $\tau_{\mathrm{p}}$, and the geometries (spacings and aperture 
sizes) of the additional electrodes were all varied by the genetic algorithm, in order to find a configuration with a large spread of arrival times for a given ion cloud, as well as good velocity resolution both along the time-of-flight axis and in the plane of the detector. This was repeated for a range of $m / z$ and kinetic energy values, and resulted in an experimental geometry for our spectrometer that achieved high performance for a range of initial ion conditions, whilst only needing to vary $\tau_{\mathrm{p}}$ and the pulsed voltage. This geometry is shown in detail in Figure 2 - highlighting the additional ion optics added to the spectrometer's previous DC slicing optics.

To characterize the velocity mapping performance of this optimized geometry, we simulated an ensemble of $\mathrm{CO}^{+}$ions (with $0.2 \mathrm{eV}$ kinetic energy) for a reasonably large cylindrical interaction region with $4 \mathrm{~mm}$ length along the laser propagation direction, and $0.4 \mathrm{~mm}$ diameter perpendicular to this. The velocity resolution (in the detector plane) predicted for the DC slicing configuration was $0.2 \%$, whilst the PEISI configuration gave a value of $0.6 \%$, with a $3 \mathrm{kV}$ pulsed voltage. While this indicates a slightly poorer velocity focussing for the PEISI approach, we note that the predicted velocity resolution is still better than that typically attainable experimentally. Factors such as velocity spread of the molecular beam, fragment recoil following ionization and the spatial resolution of the detector generally result in a poorer experimental velocity resolution. The predicted velocity resolution was approximately constant over a wide range of ion kinetic energies $(0.05-1 \mathrm{eV})$, indicating minimal chromatic abberations.

Figure 3 shows the achievable time-of-flight spread, $\Delta t$, with this optimized geometry for a range of ion masses, each with a fixed kinetic energy of $0.2 \mathrm{eV}$. In each case, the repeller voltage was fixed to $1 \mathrm{kV}$ and the optimal $\tau_{\mathrm{p}}$ was found through a brute force search. The voltage of the pulsed plate was $3 \mathrm{kV}$. It can be seen that employing PEISI results in significantly greater temporal stretching for all masses (by a constant factor of approximately 9). Of particular note is the indication that PEISI could be used for the slice imaging of very light (i.e. low momentum) photofragments such as $\mathrm{H}^{+}$. While slice imaging of $\mathrm{H}^{+}$fragments has been demonstrated in delayed extraction experiments [60], this is typically challenging for DC slicing setups [61].

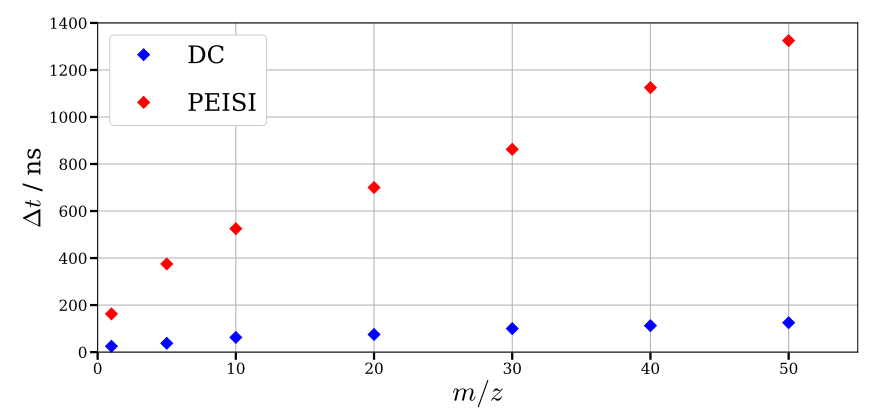

Figure 3. Photofragment temporal stretch, $\Delta t$, as a function of ion mass for simulated singly-charged ions with a kinetic energy of $0.2 \mathrm{eV}$, using a repeller voltage of $1 \mathrm{kV}$ and a pulsed voltage of $3 \mathrm{kV}$.

\section{Experimental methods}

Experiments were carried out using a velocity map imaging spectrometer which has been described in detail previously [21]. Two additional electrodes were added to a four electrode DC slicing assembly: a pulsed cylindrical plate and a grounded grid (Goodfellow electro-formed Ni mesh, 500 wires/inch). The resulting assembly employs 
the geometry displayed in Figure 2(b). In the PEISI experiments, the pulsed plate is initially held at ground, with a large positive voltage applied at a pulse time $\tau_{\mathrm{p}}$ using a fast Behlke switch, with a rise time of $c a .50 \mathrm{~ns}$. The optimum $\tau_{\mathrm{p}}$ for a given fragment was determined using SIMION, and verified experimentally.

A mixture of $5 \%$ OCS in helium (BOC UK) at a stagnation pressure of 1 bar was expanded as a pulsed molecular beam through a Series 9 General Valve. The resultant molecular beam was collimated through a $1 \mathrm{~mm}$ skimmer before passing through a $1 \mathrm{~mm}$ aperture in the repeller plate. Here, molecules were intersected perpendicularly by a UV laser pulse, which induced photodissociation and subsequent REMPI of the nascent $\mathrm{CO}$ or $\mathrm{S}\left({ }^{1} \mathrm{D}_{2}\right)$ fragments. Tunable laser light around $230 \mathrm{~nm}$ was generated using the frequency doubled output of a dye laser (Sirah CSTR-G-24) operating with Coumarin 460 laser dye. This was pumped by the third harmonic of a pulsed Nd:YAG laser (Continuum Powerlite Precision II).

Resultant ions were velocity mapped by the electrode assembly and accelerated along a $620 \mathrm{~mm}$ flight tube towards a position-sensitive detector consisting of a pair of chevron-stacked MCPs and a $75 \mathrm{~mm}$ diameter P47 phosphor screen. The MCPs were operated with a $1.90 \mathrm{kV}$ potential difference between the front and back faces, with a $4 \mathrm{kV}$ potential between the back MCP and the scintillator. The flashes of light caused by the ion impacts were imaged using a Pixel Imaging Mass Spectrometry (PImMS) camera equipped with a PImMS2 sensor [62-64]. This comprises a $324 \times 324$ pixel array, that was set to record arrival times $(t)$ of incident photos to a precision of $25 \mathrm{~ns}$ (hereafter referred to as a timebin). This fast-timestamping ability allowed for the recording of many time-sliced images through the measured Newton spheres, from which the three-dimensional velocity distribution can be determined.

Ion events typically illuminate multiple PImMS pixels, across multiple timebins. To account for this, ion events were identified through a three-dimensional nearestneighbour search and centroided in time and space as described previously [21, 65-67]. Smaller clusters $(\leq 4$ pixels) were excluded in order to further improve the spatiotemporal resolution of the data. Images were typically four-fold symmetrised in order to improve the signal-to-noise ratio.

\section{Results \& discussion}

To experimentally validate the PEISI technique, the one colour photodissociation and REMPI of OCS was studied at around $230 \mathrm{~nm}$. The UV photodissociation of OCS has been well studied previously [35, 42-46, 48-55], and is known to occur following excitation to the $1^{1} \mathrm{~A}^{\prime \prime}$ and $2^{1} \mathrm{~A}^{\prime}$ states. A significantly bent structure in these excited states generates a high torque on the resultant fragments to give highly rotationally excited CO. A bimodal rotational distribution of the $\mathrm{CO}$ fragment arises primarily as a result of multiple dissociation pathways. Lower rotational state products arise from dissociation on both surfaces, whilst those formed in high rotational states are produced following non-adiabatic transitions to the $1^{1} \mathrm{~A}^{\prime}$ ground state. $\mathrm{CO}$ and $\mathrm{S}\left({ }^{1} \mathrm{D}_{2}\right)$ photoproducts can both be ionized in $(2+1)$ REMPI schemes around $230 \mathrm{~nm}$, via transitions to the $\mathrm{B}^{1} \Sigma^{+}$and $\left({ }^{2} \mathrm{D}_{3 / 2,5 / 2}\right) 7 \mathrm{f}$ states, respectively $[43,68]$. The resulting REMPI spectra are shown in Figure 4, which displays the characteristic bimodal distribution of $\mathrm{CO}$ rotational states. 


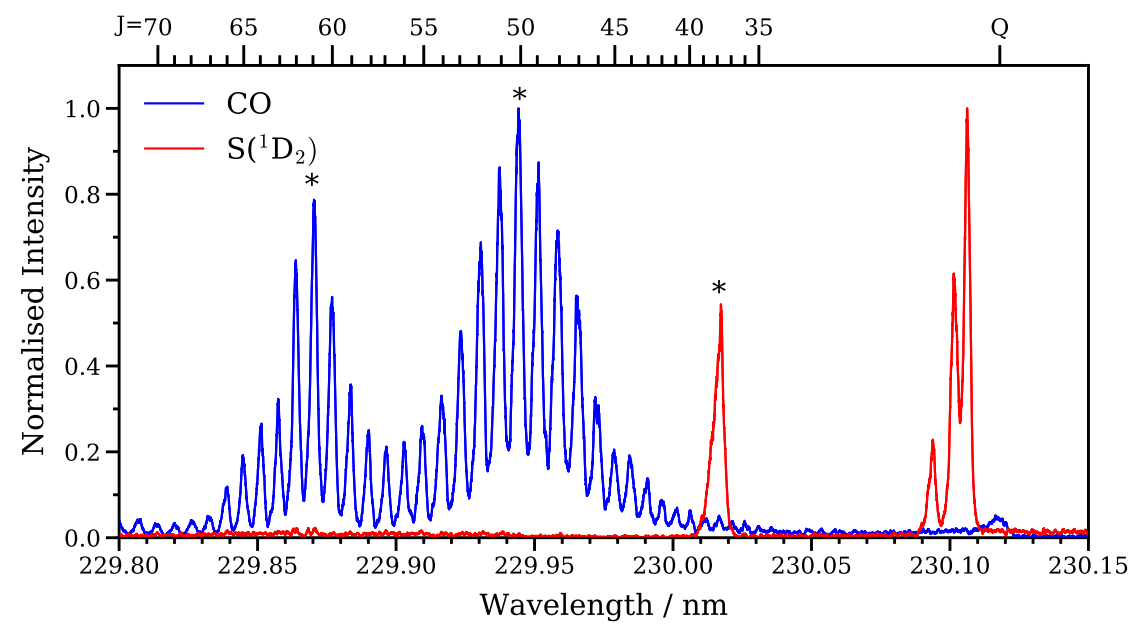

Figure 4. (2+1) REMPI spectra of the $\mathrm{CO}$ (blue line) and $\mathrm{S}\left({ }^{1} \mathrm{D}_{2}\right)$ (red line) photofragments following OCS photodissociation. Asterisks denote peaks for which images are displayed in this work.

\subsection{Sliced and three-dimensional imaging}

Figure 5 compares central slice images and velocity distributions recorded for the $\mathrm{CO}(J=50)$ photofragment using the DC slicing and PEISI techniques, both employing a repeller voltage of $0.50 \mathrm{kV}$, with a $3 \mathrm{kV}$ pulsed voltage in the PEISI case. The 'tail' in the velocity distribution as a result of the out of plane velocity contributions is not present in the PEISI case, indicative of imaging a significantly narrower slice of the three-dimensional velocity distribution. This leads to a modest improvement in the observed velocity resolution. The slightly faster ring arising from dissociation of OCS molecules with a quantum of excitation in the bending vibrational mode [49] is also more clearly resolved than in the DC case. We are unable to resolve the small contribution from dissociation of $\mathrm{OC}^{34} \mathrm{~S}[8]$. There is no evidence of any non-circularity or other distortions in the recorded PEISI image. It should be noted that the observed velocity resolution of $\approx 3 \%$ is most likely limited by experimental factors associated with the instrument employed (e.g., by the limited pixel array of the camera, transverse velocity spread of the molecular beam and the effect of electron recoil following ionization), with the ultimate resolution of the technique predicted by simulations to be far greater.

By modifying the delay of the pulsed voltage, conditions can be optimized for a particular $m / z$ value or a small range of values. Figure 6 displays time-of-flight spectra and sliced ion images for the $\mathrm{S}\left({ }^{1} \mathrm{D}_{2}\right)$ and $\mathrm{CO}(J=38)$ photofragments recorded around $230.01 \mathrm{~nm}$. In panel (a) no pulsed voltage is applied, in (b) a pulse of $3 \mathrm{kV}$ is applied at $\tau_{\mathrm{p}}$ of $3.10 \mu \mathrm{s}$ (optimized for the $\mathrm{S}^{+}$ion), in (c) the pulse is applied at $\tau_{\mathrm{p}}$ of $2.92 \mu \mathrm{s}$ (optimized for the $\mathrm{CO}^{+}$ion). These timings were predicted using SIMION and verified experimentally, and a repeller voltage of $1 \mathrm{kV}$ was used in all cases. Here, significant broadening of the time of flight peak(s) and the resulting improved resolution in the central slice images can be seen clearly. In the $\mathrm{S}\left({ }^{1} \mathrm{D}_{2}\right)$ image, more fine-structure can be resolved when the PEISI technique is used to temporally stretch the ion cloud. The observation of many rings within the lower radius feature in panel (b) arises from $\mathrm{S}\left({ }^{1} \mathrm{D}_{2}\right)$ fragments paired with different rotational states of the $\mathrm{CO}$ cofragment (see Figure 4 for a representative $\mathrm{CO}$ rotational distribution). In particular, features at low radius/velocity are sharper than in the DC case. This is due to the fact that the 
a) DC Slicing

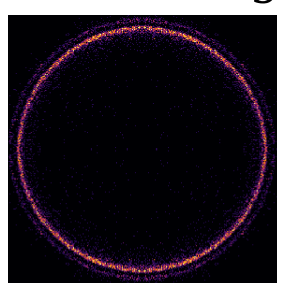

b) PEISI

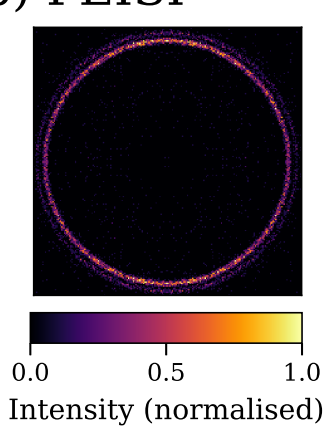

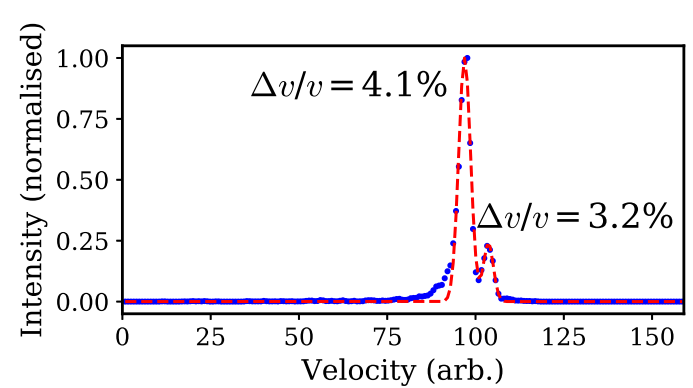

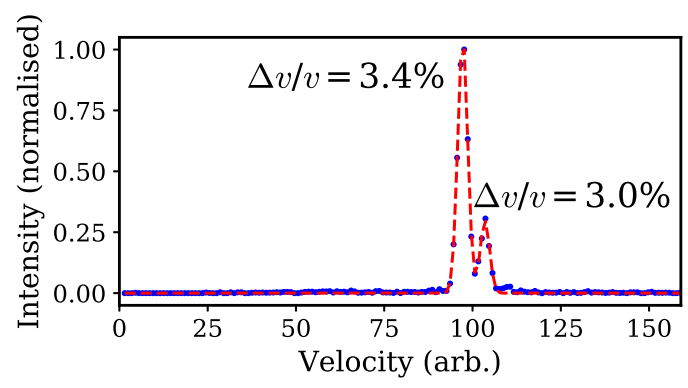

Figure 5. Left: comparison of sliced images of $\mathrm{CO}(J=50)$ under (a) DC slicing and (b) PEISI conditions. Right: corresponding velocity distributions (blue points) with velocity resolutions $(\Delta v / v)$, with $\Delta v$ given by the full width at half maximum (FWHM) obtained from Gaussian fits (red dashed line).

imaged central slice represents a far larger solid angle of the three-dimensional velocity distribution for slower ions in comparison to the fast ions, and so the better slicing resolution of the PEISI approach is of greatest importance here. This can be seen in comparing the kinetic energy distributions of the $S\left({ }^{1} D_{2}\right)$ fragment extracted from the images in Figure 6(a) and (b), which are plotted in Figure 7. Evenly spaced peaks, corresponding to different rotational states of the $\mathrm{CO}$ cofragment can be more clearly distinguished in the PEISI case. The photofragment anisotropy parameter, $\beta$, can be extracted for each feature, which are also plotted. For the majority of the resolved peaks, a $\beta$ of around 1.1 is observed, in reasonable agreement with previous studies $[52,69,70]$. This rapidly changes for the two lowest radius features, which have far more isotropic angular distributions. A full description of the dynamics responsible for this is beyond the scope of the current manuscript, however such effects have been recently explored in detail by Janssen and coworkers [54, 55]. At higher radius, rings corresponding to individual CO rotational states can no longer be resolved. However, the observed $\beta$ of $0.33 \pm 0.02$ averaged over the high $\mathrm{S}$ velocity feature is in good agreement with literature values, such as the 0.24 and 0.71 reported by Janssen and coworkers for OCS molecules with zero or one quanta of excitation in the bending mode [49].

While the PEISI technique offers significant benefits for slice imaging applications, perhaps the most powerful demonstration of the technique arises from its use in full three-dimensional imaging, permitted by the use of a fast time-stamping detector. Several sets of full three-dimensional data (presented as successive time slices through recorded Newton sphere(s)) can be found in the online Supplementary Information. Figure 8 shows an example set of ion images for the $\mathrm{CO}(J=50)$ photofragment, using voltages of $1 \mathrm{kV}$ and $3 \mathrm{kV}$ on the repeller and pulsed plates, respectively. The series of 


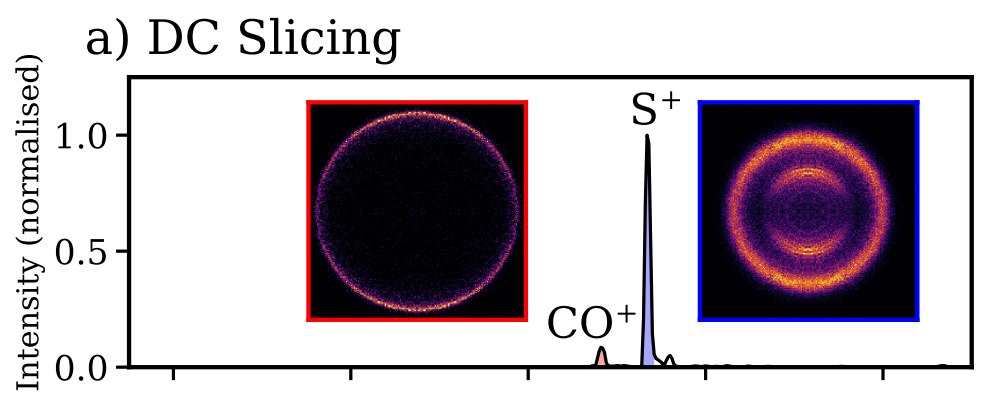

b) Pulsed $\mathrm{S}^{+}$
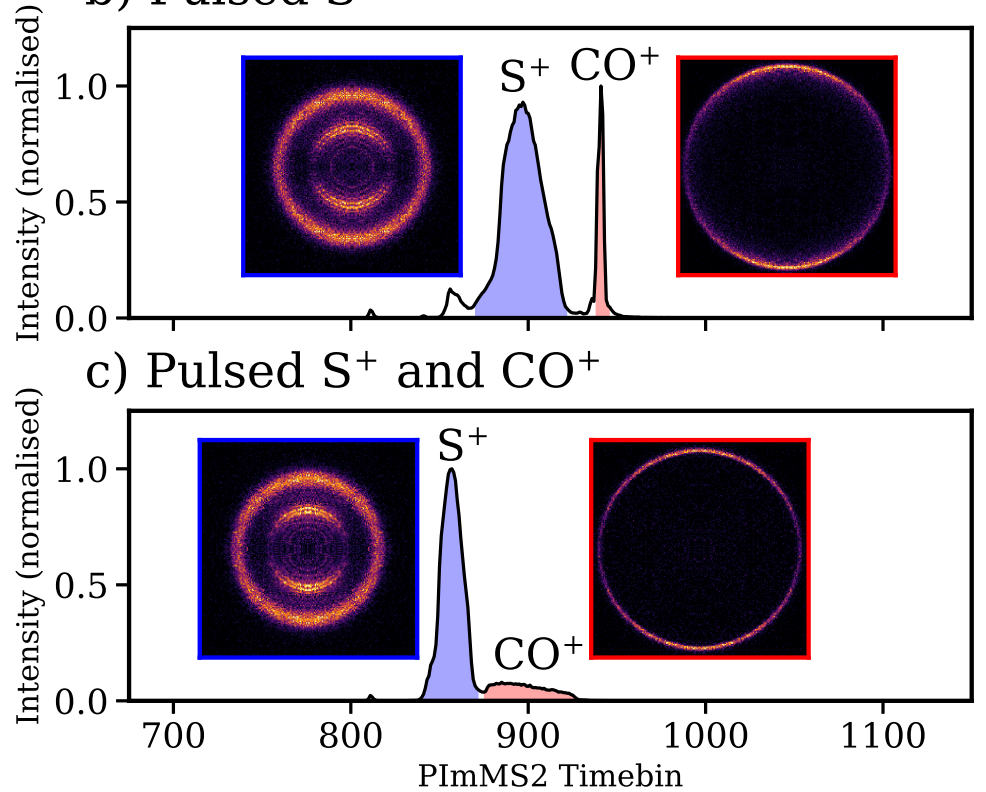

Figure 6. PImMS time-of-flight spectra and accompanying central slice images for the $\mathrm{S}\left({ }^{1} \mathrm{D}_{2}\right)$ and $\mathrm{CO}(J=$ 38) photofragments under differing experimental conditions: (a) DC slicing, (b) a pulsed voltage applied at $3.10 \mu \mathrm{s}$ (optimized for the $\mathrm{S}^{+}$ion), and (c) a pulsed voltage applied at $2.92 \mu \mathrm{s}$ (optimized for the $\mathrm{CO}^{+}$ion).

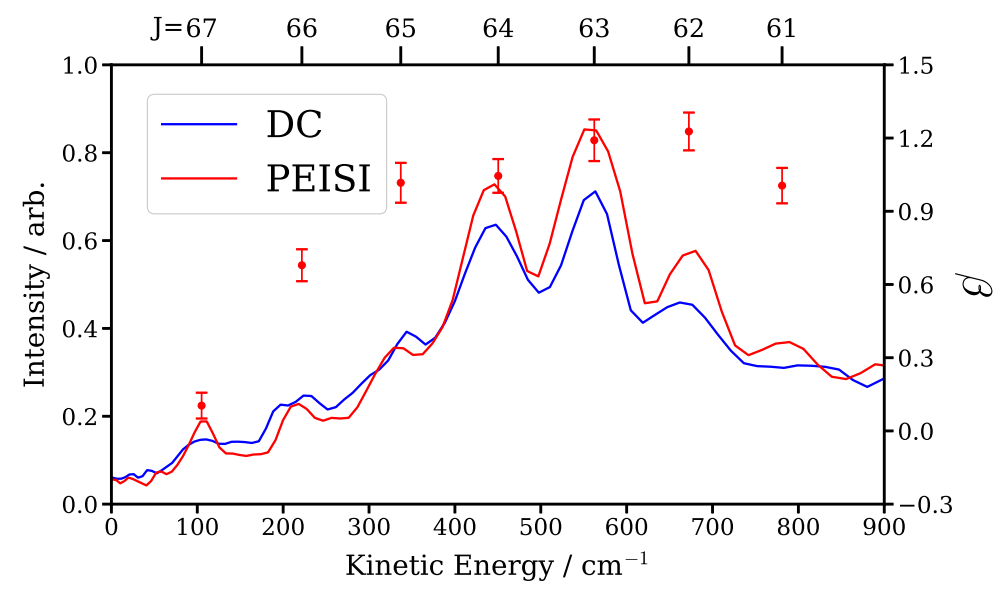

Figure 7. Comparison of the kinetic energy distributions of the low radius feature in the $\left.\mathrm{S}^{1} \mathrm{D}_{2}\right)$ images from panels (a) and (b) of Figure 6. Anisotropy parameters, $\beta$, for each peak in the PEISI image are plotted as red markers, with standard fit errors. 
time-sliced images each represent a thin slice through the three-dimensional ion cloud, along the time-of-flight axis.
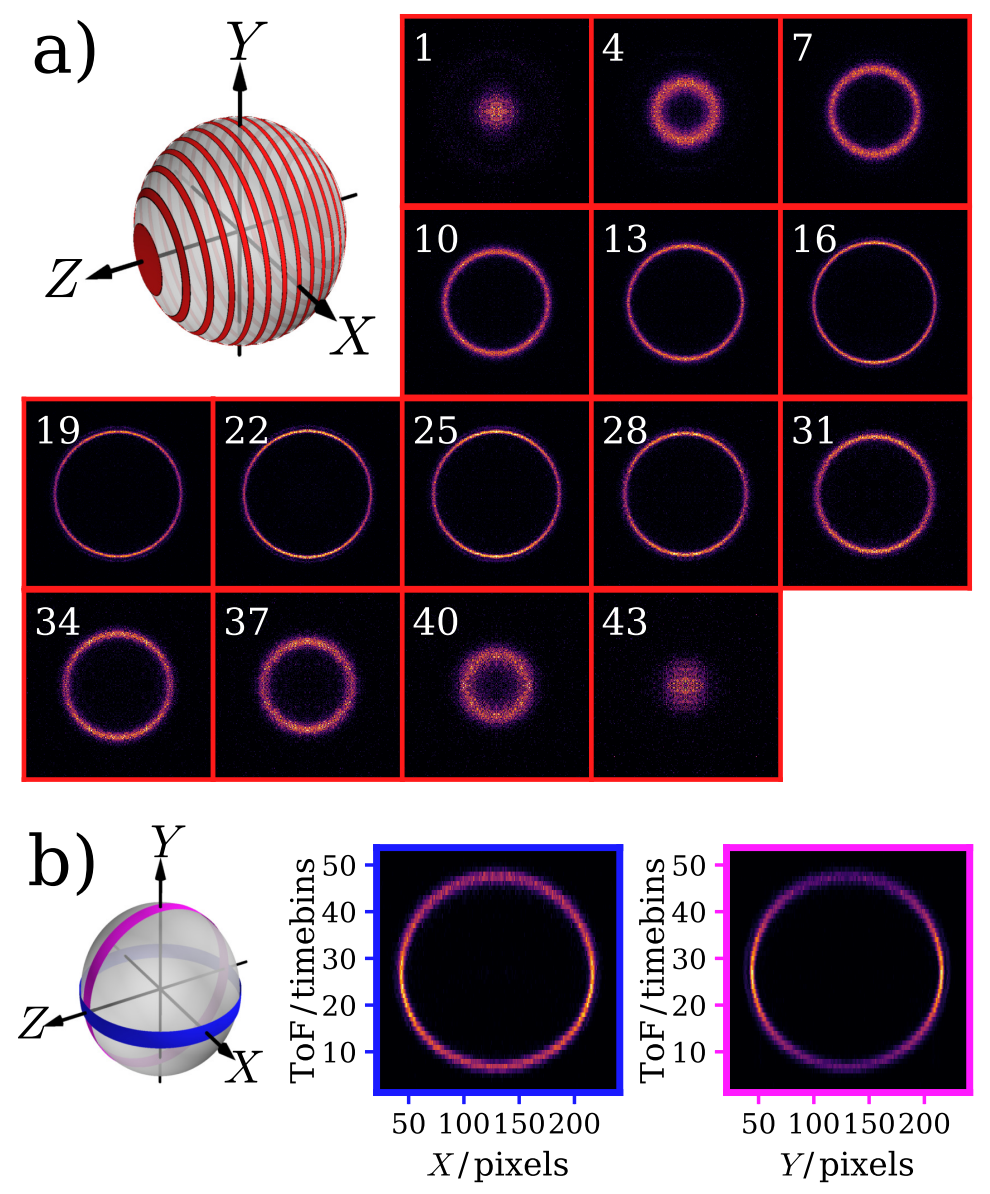

Figure 8. (a) Time-sliced images of $\mathrm{CO}(J=50)$ following OCS photodissociation, each corresponding to one $25 \mathrm{~ns}$ timebin. Each is labelled by its relative PImMS timebin through the $\mathrm{CO}^{+}$time-of-flight peak. The intensity of each image is normalised separately. (b) Central slice images in the (ToF, $X)$ and (ToF, $Y$ ) planes, as described in the text. Representations of how these images related to the photofragment Newton spheres in the laboratory frame are also shown. Here, the laser propagates along the $X$ axis and is polarized along the $Y$ axis. The spectrometer time-of-flight axis lies along the $Z$ axis.

As one looks at images moving further away from the central slice, the corresponding solid angle of the Newton sphere being projected necessarily increases. As a result, in order to image a significant portion of the Newton sphere with high-resolution, the increased stretching of the ion cloud by the PEISI technique becomes essential, given the time resolution of the detector. As clearly demonstrated in Figure 8(a), sliced images throughout almost the entire Newton sphere (1.125 $\mu \mathrm{s}$, or $4525 \mathrm{~ns}$ PImMS timebins) remain relatively sharp. For reference, our previous work employing a DC slicing approach under otherwise similar conditions yielded images spanning $138 \mathrm{~ns}$ (11 12.5 ns timebins) in arrival time [21]. The sharp three-dimensional velocity resolution can be further verified by viewing slices through the Newton sphere along the time-of-flight axis, as shown in Figures 8(b) for slices in the (ToF, $X)$ and (ToF, $Y$ ) planes. The spatial widths of these slices in the $Y$ and $X$ directions are 17 pixels, approximately $10 \%$ of the full ring. The recorded velocity distributions in these planes out of the detector remain well resolved due to the temporal stretching afforded by the PEISI technique, 
and so the recorded velocities in all three-dimensions may be usefully analysed. It should be noted that the images shown in panels (b) are slightly asymmetric along the time-of-flight axis. This is due to some non-linearity in the relationship between the initial ion velocity along this axis, and the ion arrival time at the detector. This is reproduced by ion trajectory calculations, and can be taken into account to extract the three-dimensional ion velocities (including weighting by an appropriate geometric Jacobian). If operating under low repeller voltages $(<1 \mathrm{kV})$, the detection efficiency of the ions was found to depend on their final time-of-flight (as a result of different impact velocities onto the detector). This variable detection efficiency must also be accounted for when analyzing the data, or the experiment should be operated at sufficiently high repeller potentials to ensure the detection efficiency of all ions is saturated.

It can also be seen in Figure 8b) that the measured three-dimensional velocity distributions are sharpest in the plane of the detector, and are less well-resolved for greater velocities along the time-of-flight axis. At the poles of the three-dimensional velocity distribution along the time-of-flight axis, the observed velocity resolution is decreased to approximately 10\%. Perpendicular to this, a velocity resolution of approximately $3 \%$ is observed, as shown in Figure 5. We ascribe this degradation of resolution to the molecular beam having a greater velocity spread in this axis (longitudinal) than in the detector plane (transverse). Given the CO recoil velocity of $\sim 1350 \mathrm{~ms}^{-1}$, the observed resolution of approximately $10 \%$ is consistent with a 5-10\% FWHM longitudinal velocity spread of our $\sim 1475 \mathrm{~ms}^{-1}$ molecular beam [71]. In future work, we anticipate it will be beneficial to account for the effects of the longitudinal velocity spread of the molecular beam when fitting recorded three-dimensional velocity distributions.

\subsection{Extraction of full three-dimensional photofragment angular distributions}

Measuring an entire three-dimensional velocity distribution, as opposed to a planar projection presents clear advantages in experimental geometries lacking an axis of cylindrical symmetry in the plane of the detector, for which the inverse Abel transform is not appropriate. Such experiments include crossed molecular beam scattering, and photofragment angular momentum polarization experiments, employing non-colinear pump and pulse laser polarizations. Here, we demonstrate that three-dimensional velocity distributions, and thus photofragment anisotropy parameters can be reliably extracted from a set of PEISI imaging data directly, making no assumptions about the symmetry of the system, nor through the fitting of the data to any basis set. In terms of potential applications to studies of photofragment angular polarization, these results suggest desired alignment parameters could be extracted from measurements in a single experimental geometry, circumventing the need to record data with multiple geometries of the polarizations of pump and probe pulses.

Generally, a photofragment angular distribution, $f(\theta, \phi)$ may be conveniently expressed as an expansion in modified spherical harmonic functions [72-74], $C_{k q}(\theta, \phi)$ :

$$
f(\theta, \phi)=\sum_{k=0}^{\infty} \sum_{q=-k}^{k} m_{k q} C_{k q}(\theta, \phi)
$$

where the expansion coefficients, $m_{k q}$ may be calculated from the angular distribution 
by:

$$
m_{k q}=\frac{2 k+1}{4 \pi} \int_{\phi=0}^{2 \pi} \int_{\theta=0}^{\pi} f(\theta, \phi) C_{k q}^{*}(\theta, \phi) \sin \theta \mathrm{d} \theta \mathrm{d} \phi
$$

In general, the $m_{k q}$ coefficients may be complex, and so these coefficient can be transformed into real parameters, $m_{q \pm}^{\{k\}}$, using Hertel-Stoll notation [75-77].

In order to evaluate the integrals in Equation (1), the arrival time $(t)$ and pixel position $(x, y)$ of a centroided ion event detected by the PImMS camera must be transformed into three-dimensional velocity space. This was done using SIMION ion trajectory simulations, accounting for the specific experimental parameters. In all test cases, these ion trajectory calculations were found to accurately reproduce the experimental observations. Following transformation of the data into velocity coordinates, the photoion angular distribution $f(\theta, \phi)$ over a given velocity range is obtained, and the integrals in Equation (1) can be directly evaluated through Monte Carlo integration. As a result, the full three-dimensional velocity distribution can now be expressed as a sum of modified spherical harmonics, with weightings given by the extracted polarization parameters. This procedure is in principle applicable to any photodissociation process, regardless of the symmetry of the system.

In our experimental system, the photodissociation and subsequent $(2+1)$ REMPI by a single $\sim 230 \mathrm{~nm}$ laser pulse, the resulting photoion angular distribution must possess cylindrical symmetry. However, to test the above procedure of spherical harmonics expansion in conditions where the inverse Abel transform of the two-dimensional projection may not be performed, the polarization vector of the light was rotated out of the plane of the two-dimensional detector using a Rochon polarizer prior to focusing into the spectrometer's interaction region. The angle of rotation $\alpha$ was nominally $20^{\circ}$. Figure 9(a) shows the resulting three-dimensional ion imaging data for the $J=61$ state of $\mathrm{CO}$, which is known to exhibit a predominantly parallel angular distribution [49]. The schematic in Figure 9(b) clarifies the direction of the polarization axis of the laser light, and defines the coordinate system used in the analysis. The effect of the rotated laser polarization can be clearly seen in the time-sliced images in which the ions arriving at earlier timebins (corresponding to negative initial velocity in the $Z$ direction) have a pronounced up/down asymmetry, which is reversed for later timebins. This can be further explored through an intensity plot of angles $\theta$ and $\phi$ following transformation of the raw data to lab-frame spherical coordinates, shown in panel (c). The lack of cylindrical symmetry in this coordinate system can be seen in the $\theta$ (angle in the plane of the detector) dependence of the $\phi$ distribution. Ions with $\phi<90^{\circ}$ have preference for $\theta$ values around $0 / 360^{\circ}$, whereas ions with $\phi>90^{\circ}$ have a preference for $\theta$ around $180^{\circ}$. It can also be seen that the $\phi$ maxima for these two regions of intensity in $\theta$ are shifted from $\phi=90^{\circ}$ by approximately $20^{\circ}$, the value of $\alpha$.

To determine the exact value of $\alpha$ in this experiment, the three-dimensional laboratory velocity distribution was transformed into a molecular frame velocity distribution, assuming a range of $\alpha$ values. Due to the cylindrical symmetry of the velocity distribution about the polarization axis ( $z$ in these molecular frame coordinates), the resulting distribution should be independent of the angle in the plane perpendicular to this axis, if converted correctly. Thus, the value of $\alpha$ that minimized the standard deviation of the velocity distribution in this angular coordinate was assumed to be the 'true' experimental value. This method yielded an $\alpha$ of $24^{\circ}$, close to the nominal value of $20^{\circ}$ set on the Rochon polarizer. 


\section{a)}

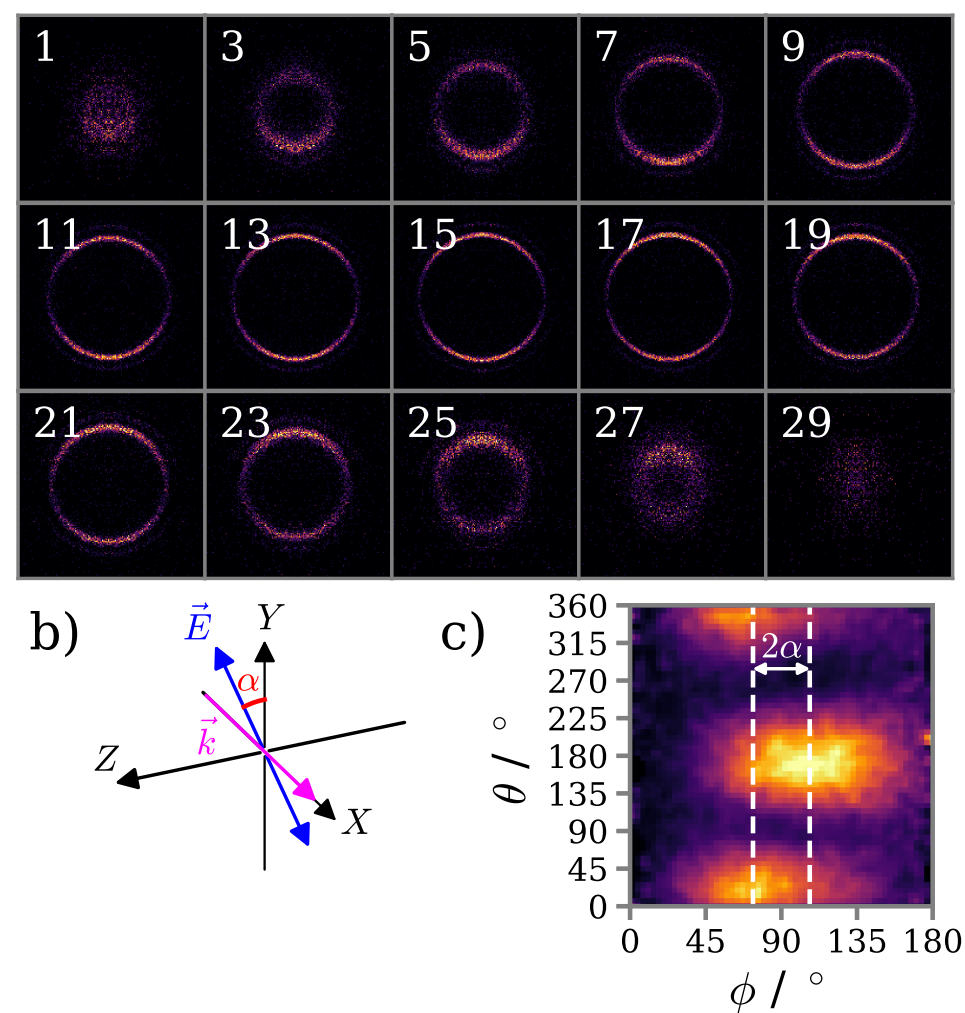

Figure 9. (a) Series of time-sliced images of $\mathrm{CO}(J=61)$ following OCS photodissociation, each corresponding to one $25 \mathrm{~ns}$ timebin. Each time-sliced image is labelled by its relative PImMS timebin through the $\mathrm{CO}^{+}$time-offlight peak. The intensity of each image is normalised separately. (b) Schematic of the experimental coordinate system, displaying the rotated polarization axis of the laser, which no longer coincides with the laboratory $Y$ axis. The laser propagates along the $X$ axis, the detector lies in the $(X, Y)$ plane (c) Three-dimensional angular distribution in the laboratory frame extracted from the data in (a)

Upon rotation of the recorded three-dimensional velocity distribution by $\alpha$ into a new coordinate system, the expansion coefficients may be calculated by evaluating Equation (2), with the required integrals performed using a Monte-Carlo approach. Table 1 displays the $m_{q \pm}^{\{k\}}$ parameters extracted from this procedure. As expected for a single photon dissociation with no alignment effects, $m_{0}^{\{2\}}(\equiv \beta / 2)$ is the only significant contribution, taking a value of 0.716 . For comparison, extracted parameters from experiments without the rotation of the laser polarization are also shown. This was done by fitting the angular distribution of the recorded central slice image, as well as through Abel inversion of the entire two-dimensional projection using the pBasex algorithm [6]. In all cases, good agreement is observed. This further indicates that direct expansion of the three-dimensional data in spherical harmonics is an effective way to extract these physically meaningful parameters, and can be done without fitting of the data, and with no restrictions on the velocity distribution's symmetry. In all cases, the observed $\beta$ values $\left(\equiv 2 m_{0}^{\{2\}}\right.$ ) are slightly lower than the literature value of 1.65 observed by van den Brom et al. [49]. However, in their experiment, OCS molecules in the $\left(\nu_{2} \mid J M=10\right)$ ro-vibrational state were selected using a hexapole, and so perfect agreement is not to be expected. 


\begin{tabular}{|c|c|c|c|c|}
\hline$k$ & $q_{ \pm}$ & $3 \mathrm{D}$ imaging & Sliced & pBasex \\
\hline 1 & $1-$ & $0.012 \pm 0.001$ & - & - \\
1 & 0 & $0.004 \pm 0.009$ & - & - \\
1 & $1+$ & $-0.040 \pm 0.002$ & - & - \\
2 & $2-$ & $-0.007 \pm 0.001$ & - & - \\
2 & $1-$ & $0.003 \pm 0.001$ & - & - \\
2 & 0 & $0.716 \pm 0.001$ & $0.713 \pm 0.044$ & $0.691 \pm 0.040$ \\
2 & $1+$ & $0.011 \pm 0.003$ & - & - \\
2 & $2+$ & $0.060 \pm 0.004$ & - & - \\
4 & 0 & $0.002 \pm 0.003$ & $0.056 \pm 0.037$ & $-0.014 \pm 0.036$ \\
\hline
\end{tabular}

Table 1. Comparison of extracted angular distribution parameters, $m_{q \pm}^{\{k\}}$. The column labelled '3D imaging' refers to the coefficients obtained from spherical harmonic expansion of the rotated 3D Newton sphere, quoted with the $95 \%$ confidence interval of the Monte Carlo integration. 'Sliced' refers to fitting of the angular distribution of central slice images of a reference data set without rotation of the laser polarization axis, quoted with the standard fit error. 'pBasex' refers to Abel inversion of the reference two-dimensional projection using the pBasex algorithm [6]. Each quadrant of the reference image was inverted separately, with the mean value quoted with an error given by the standard deviation.

\section{Conclusions \& outlook}

We have presented an experimental modification to a typical velocity map ion imaging spectrometer to greatly improve performance in sliced and three-dimensional imaging experiments. This relies upon inversion and subsequent stretching of the Newton sphere along the time-of-flight axis through applying a pulsed voltage in the postextraction region. The improvements in slicing resolution have been experimentally verified on a well-studied system. An advantage of this experimental approach has been highlighted through the extraction of a set of photofragment anisotropy parameters in an experimental geometry where the inverse Abel transform could not be used, by expanding the three-dimensional ion image in spherical harmonics.

As mentioned, this technique would be useful in crossed molecular beams experiments to record three-dimensional bimolecular scattering distributions, or to photolysis experiments studying photofragment angular momentum polarization. In principle, high fidelity three-dimensional imaging, as afforded by the PEISI technique, can circumvent the need for multiple experimental geometries of pump and probe pulse polarizations. Development of data analysis techniques to account for the effects of finite slice width $[8,19]$ and the velocity spread of the molecular beam on the recorded threedimensional velocity distributions would be of great interest. Application to multi-mass Coulomb explosion imaging experiments [67, 78-81] for high-resolution 3D covariance imaging of correlated ion momenta (as has been recently demonstrated in a DC slicing experiment [22]) is ongoing. Currently, the technique is only valid for a rather narrow mass-to-charge range. Development of different electrode geometries and/or more complex time-dependent ion optical potentials [82] could expand this range.

\section{Acknowledgements}

We are grateful for the support of the UK EPSRC (M. Br. - Programme Grant No. EP/L005913/1; M. Bu. Early Career Fellowship EP/S028617/1), and the STFC (PN- 
PAS award and mini-IPS grant No. ST/J002895/1). F.A. thanks the ESPRC and Magdalen College, Oxford for their support.

\section{References}

[1] D.W. Chandler and P.L. Houston, J. Chem. Phys. 87 (2), 1445-1447 (1987).

[2] A.T.J.B. Eppink and D.H. Parker, Rev. Sci. Instrum. 68 (9), 3477-3484 (1997).

[3] M.N. Ashfold, N.H. Nahler, A.J. Orr-Ewing, O.P. Vieuxmaire, R.L. Toomes, T.N. Kitsopoulos, I.A. Garcia, D.A. Chestakov, S.M. Wu and D.H. Parker, Phys. Chem. Chem. Phys. 8 (1), 26-53 (2006).

[4] A.G. Suits, Rev. Sci. Instrum. 89 (11), 111101 (2018).

[5] V. Dribinski, A. Ossadtchi, V.A. Mandelshtam and H. Reisler, Rev. Sci. Instrum. 73 (7), 2634 (2002).

[6] G.A. Garcia, L. Nahon and I. Powis, Rev. Sci. Instrum. 75 (11), 4989-4996 (2004).

[7] B. Dick, Phys. Chem. Chem. Phys. 16 (2), 570-580 (2014).

[8] J.O. Thompson, C. Amarasinghe, C.D. Foley and A.G. Suits, J. Chem. Phys. 147 (1) (2017).

[9] B. Dick, Phys. Chem. Chem. Phys. 21 (35), 19499-19512 (2019).

[10] M.J. Bass, M. Brouard, A.P. Clark and C. Vallance, J. Chem. Phys. 117 (19), 8723-8735 (2002).

[11] M.J. Bass, M. Brouard, A.P. Clark, C. Vallance and B. Martínez-Haya, Phys. Chem. Chem. Phys. 5 (5), 856-864 (2003).

[12] A.G. Suits and O.S. Vasyutinskii, Chem. Rev. 108 (9), 3706-3746 (2008).

[13] X. Yang, Annu. Rev. Phys. Chem. 58 (1), 433-459 (2007).

[14] S.J. Greaves, R.A. Rose and A.J. Orr-Ewing, Phys. Chem. Chem. Phys. 12 (32), 9129 $9143(2010)$.

[15] C.R. Gebhardt, T.P. Rakitzis, P.C. Samartzis, V. Ladopoulos and T.N. Kitsopoulos, Rev. Sci. Instrum. 72 (10), 3848-3853 (2001).

[16] D. Townsend, M.P. Minitti and A.G. Suits, Rev. Sci. Instrum. 74 (4), 2530-2539 (2003).

[17] J.J. Lin, J. Zhou, W. Shiu and K. Liu, Rev. Sci. Instrum. 74 (4), 2495-2500 (2003).

[18] K. Mizuse, R. Fujimoto and Y. Ohshima, Rev. Sci. Instrum. 90 (10), 103107 (2019).

[19] J.O. Thompson, C. Amarasinghe, C.D. Foley, N. Rombes, Z. Gao, S.N. Vogels, S.Y. Van De Meerakker and A.G. Suits, J. Chem. Phys. 147 (7) (2017).

[20] D.J. Harding, J. Neugebohren, H. Hahn, D.J. Auerbach, T.N. Kitsopoulos and A.M. Wodtke, J. Chem. Phys. 147 (1) (2017).

[21] K. Amini, S. Blake, M. Brouard, M.B. Burt, E. Halford, A. Lauer, C.S. Slater, J.W. Lee and C. Vallance, Rev. Sci. Instrum. 86 (10), 103113 (2015).

[22] J.W.L. Lee, H. Köckert, D. Heathcote, D. Popat, R.T. Chapman, G. Karras, P. Majchrzak, E. Springate and C. Vallance, Commun. Chem. 3 (1), 72 (2020).

[23] R. Forbes, F. Allum, S. Bari, R. Boll, K. Borne, M. Brouard, H. Philip, N. Ekanayake, B. Erk, A.J. Howard, P. Johnsson, W. Jason, L. Lee, B. Manschwetus, R. Mason, C. Passow, J. Peschel, D.E. Rivas, R. Aljoscha, A. Rouzée, C. Vallance, F. Ziaee, D. Rolles and M. Burt, J. Phys. B At. Mol. Phys. (2020).

[24] J.H. Jungmann, A. Gijsbertsen, J. Visser, J. Visschers, R.M.A. Heeren and M.J.J. Vrakking, Rev. Sci. Instrum. 81 (10), 103112 (2010).

[25] J. Long, F.J. Furch, J. Durá, A.S. Tremsin, J. Vallerga, C.P. Schulz, A. Rouzée and M.J. Vrakking, J. Chem. Phys. 147 (1), 013919 (2017).

[26] A. Zhao, M. Van Beuzekom, B. Bouwens, D. Byelov, I. Chakaberia, C. Cheng, E. Maddox, A. Nomerotski, P. Svihra, J. Visser, V. Vrba and T. Weinacht, Rev. Sci. Instrum. 88 (11), 113104 (2017).

[27] D.A. Debrah, G.A. Stewart, G. Basnayake, A. Nomerotski, P. Svihra, S.K. Lee and W. Li, Rev. Sci. Instrum. 91 (2), 023316 (2020). 
[28] S.K. Lee, F. Cudry, Y.F. Lin, S. Lingenfelter, A.H. Winney, L. Fan and W. Li, Rev. Sci. Instrum. 85 (12), 123303 (2014).

[29] S.K. Lee, Y.F. Lin, S. Lingenfelter, L. Fan, A.H. Winney and W. Li, J. Chem. Phys. 141 (22), 221101 (2014).

[30] C. Weeraratna, C. Amarasinghe, S.K. Lee, W. Li and A.G. Suits, J. Chem. Phys. 149 (8), 084202 (2018).

[31] T.P. Rakitzis, P.C. Samartzis, R.L. Toomes, T.N. Kitsopoulos, A. Brown, G.G. BalintKurti, O.S. Vasyutinskii and J.A. Beswick, Science 300 (5627), 1936-1938 (2003).

[32] J.J. Lin, J. Zhou, W. Shiu and K. Liu, Science 300 (5621), 966-9 (2003).

[33] D. Townsend, S. Kyoung Lee and A.G. Suits, Chem. Phys. 301 (2-3), 197-208 (2004).

[34] D. Townsend, S.A. Lahankar, S.K. Lee, S.D. Chambreau, A.G. Suits, X. Zhang, J. Rheinecker, L.B. Harding and J.M. Bowman, Science 306 (5699), 1158-1161 (2004).

[35] M. Laura Lipciuc and M.H. Janssen, Phys. Chem. Chem. Phys. 8 (25), 3007-3016 (2006).

[36] W. Zhang, H. Kawamata and K. Liu, Science 325 (5938), 303-306 (2009).

[37] L. Rubio-Lago, A. García-Vela, A. Arregui, G.A. Amaral and L. Bañares, J. Chem. Phys. 131 (17), 174309 (2009).

[38] M.L. Hause, N. Herath, R. Zhu, M.C. Lin and A.G. Suits, Nat. Chem. 3 (12), 932-937 (2011).

[39] S. Hosokawa, N. Takahashi, M. Saito and Y. Haruyama, Rev. Sci. Instrum. 81 (6), 063301 (2010).

[40] N. Takahashi, Y. Adachi, M. Saito and Y. Haruyama, Nucl. Instruments Methods Phys. Res. Sect. B Beam Interact. with Mater. Atoms 315, 51-54 (2013).

[41] K. Fehre, D. Trojanowskaja, J. Gatzke, M. Kunitski, F. Trinter, S. Zeller, L.P.H. Schmidt, J. Stohner, R. Berger, A. Czasch, O. Jagutzki, T. Jahnke, R. Dörner and M.S. Schöffler, Rev. Sci. Instrum. 89 (4), 045112 (2018).

[42] M. Kawasaki, Y. Sato, K. Suto, Y. Matsumi and S.H. Wilson, Chem. Phys. Lett. 251, 67-73 (1996).

[43] R.A. Morgan, M.A. Baldwin, D. Ascenz, A.J. Orr-Ewing, M.N. Ashfold, W.J. Buma, J.B. Milan, C.R. Scheper and C.A. De Lange, Int. J. Mass Spectrom. Ion Process. 159, 1-11 (1996).

[44] H. Katayanagi, Y. Mo and T. Suzuki, Chem. Phys. Lett. 247, 571-576 (1995).

[45] Z.H. Kim, A.J. Alexander and R.N. Zare, J. Phys. Chem. A 103 (49), 10144-10148 (1999).

[46] A. Sugita, M. Mashino, M. Kawasaki, Y. Matsumi, R. Bersohn, G. Trott-Kriegeskorte and K.H. Gericke, J. Chem. Phys. 112 (16), 7095-7101 (2000).

[47] T.P. Rakitzis, P.C. Samartzis and T.N. Kitsopoulos, Phys. Rev. Lett. 87 (12), 123001 (2001).

[48] H. Katayanagi and T. Suzuki, Chem. Phys. Lett. 360 (1-2), 104-110 (2002).

[49] A.J. Van Den Brom, T.P. Rakitzis, J. Van Heyst, T.N. Kitsopoulos, S.R. Jezowski and M.H. Janssen, J. Chem. Phys. 117 (9), 4255-4263 (2002).

[50] A.J. Van Den Brom, T.P. Rakitzis and M.H. Janssen, J. Chem. Phys. 123 (16), 164313 (2005).

[51] S.K. Lee, R. Silva, S. Thamanna, O.S. Vasyutinskii and A.G. Suits, J. Chem. Phys. 125 (14), 144318 (2006).

[52] M. Brouard, A.V. Green, F. Quadrini and C. Vallance, J. Chem. Phys. 127 (8), 084304 (2007).

[53] W. Wei, C.J. Wallace, G.C. McBane and S.W. North, J. Chem. Phys. 145 (2), 24310 144318 (2016).

[54] D. Sofikitis, J. Suarez, J.A. Schmidt, T.P. Rakitzis, S.C. Farantos and M.H. Janssen, Phys. Rev. Lett. 118 (25), 253001 (2017).

[55] D. Sofikitis, J. Suarez, J.A. Schmidt, T.P. Rakitzis, S.C. Farantos and M.H. Janssen, Phys. Rev. A 98 (3), 033417 (2018).

[56] W.C. Wiley and I.H. McLaren, Rev. Sci. Instrum. 26 (12), 1150-1157 (1955).

[57] J. Aoki, H. Hazama and M. Toyoda, J. Mass Spectrom. Soc. Jpn. 59 (3), 57-61 (2011).

[58] D. Manura and D. Dahl, SIMION (R) 8.1. 
[59] M. Mitchell, An Introduction to Genetic Algorithms (MIT Press, Cambridge, MA, USA, 1998).

[60] R.L. Toomes, P.C. Samartzis, T.P. Rakitzis and T.N. Kitsopoulos, in Chem. Phys., Vol. 301, jun (, , 2004), pp. 209-212.

[61] M. Ryazanov and H. Reisler, J. Chem. Phys. 138 (14), 144201 (2013).

[62] A. Nomerotski, S. Adigun-Boaye, M. Brouard, E. Campbell, A. Clark, J. Crooks, J.J. John, A.J. Johnsen, C. Slater, R. Turchetta, C. Vallance, E. Wilman and W.H. Yuen, Nucl. Instruments Methods Phys. Res. Sect. A 633, S243-S246 (2011).

[63] A.T. Clark, J.P. Crooks, I. Sedgwick, R. Turchetta, J.W. Lee, J.J. John, E.S. Wilman, L. Hill, E. Halford, C.S. Slater, B. Winter, W.H. Yuen, S.H. Gardiner, M.L. Lipciuc, M. Brouard, A. Nomerotski and C. Vallance, J. Phys. Chem. A 116 (45), 10897-10903 (2012).

[64] J.J. John, M. Brouard, A. Clark, J. Crooks, E. Halford, L. Hill, J.W.L. Lee, A. Nomerotski, R. Pisarczyk, I. Sedgwick, C.S. Slater, R. Turchetta, C. Vallance, E. Wilman, B. Winter and W.H. Yuen, J. Instrum. 7, C08001-C08001 (2012).

[65] B.Y. Chang, R.C. Hoetzlein, J.A. Mueller, J.D. Geiser and P.L. Houston, Rev. Sci. Instrum. 69 (4), 1665-1670 (1998).

[66] C.S. Slater, Ph. D. thesis, University of Oxford, 2013.

[67] C.S. Slater, S. Blake, M. Brouard, A. Lauer, C. Vallance, J.J. John, R. Turchetta, A. Nomerotski, L. Christensen, J.H. Nielsen, M.P. Johansson and H. Stapelfeldt, Phys. Rev. A 89 (1), 011401 (2014).

[68] R.A. Morgan, A.J. Orr-Ewing, D. Ascenzi, M.N. Ashfold, W.J. Buma, C.R. Scheper and C.A. De Lange, J. Chem. Phys. 105 (6), 2141-2152 (1996).

[69] Y. Mo, H. Katayanagi and T. Suzuki, Phys. Rev. Lett. 77 (5), 830-833 (1996).

[70] T.P. Rakitzis, P.C. Samartzis and T.N. Kitsopoulos, J. Chem. Phys. 111 (23), 1041510417 (1999).

[71] G. Scoles, in .

[72] R.N. Zare and D.R. Herschbach, Proc. IEEE 51 (1), 173-182 (1963).

[73] R.N. Zare, Angular Momentum, Understanding Spatial Aspects in Chemistry and Physics (Wiley, New York, 1988).

[74] R.N. Zare, Chem. Phys. Lett. 156 (1), 1-6 (1989).

[75] J. MacEk and I.V. Hertel, J. Phys. B At. Mol. Phys. 7 (16), 2173-2188 (1974).

[76] I.V. Hertel and W. Stoll, J. Phys. B At. Mol. Phys. 7 (5), 570-582 (1974).

[77] I.V. Hertel and W. Stoll, Adv. At. Mol. Phys. 13, 113-228 (1978).

[78] M. Pitzer, M. Kunitski, A.S. Johnson, T. Jahnke, H. Sann, F. Sturm, L.P.H. Schmidt, H. Schmidt-Böcking, R. Dörner, J. Stohner, J. Kiedrowski, M. Reggelin, S. Marquardt, A. Schießer, R. Berger and M.S. Schöffler, Science 341 (6150), 1096-1100 (2013).

[79] J.D. Pickering, K. Amini, M. Brouard, M. Burt, I.J. Bush, L. Christensen, A. Lauer, J.H. Nielsen, C.S. Slater and H. Stapelfeldt, J. Chem. Phys. 144 (16), 161105-161105 (2016).

[80] M. Burt, K. Amini, J.W. Lee, L. Christiansen, R.R. Johansen, Y. Kobayashi, J.D. Pickering, C. Vallance, M. Brouard and H. Stapelfeldt, J. Chem. Phys. 148 (9), 091102 (2018).

[81] F. Allum, M. Burt, K. Amini, R. Boll, H. Köckert, P.K. Olshin, S. Bari, C. Bomme, F. Brauße, B. Cunha de Miranda, S. Düsterer, B. Erk, M. Géléoc, R. Geneaux, A.S. Gentleman, G. Goldsztejn, R. Guillemin, D.M. Holland, I. Ismail, P. Johnsson, L. Journel, J. Küpper, J. Lahl, J.W. Lee, S. Maclot, S.R. Mackenzie, B. Manschwetus, A.S. Mereshchenko, R. Mason, J. Palaudoux, M.N. Piancastelli, F. Penent, D. Rompotis, A. Rouzée, T. Ruchon, A. Rudenko, E. Savelyev, M. Simon, N. Schirmel, H. Stapelfeldt, S. Techert, O. Travnikova, S. Trippel, J.G. Underwood, C. Vallance, J. Wiese, F. Ziaee, M. Brouard, T. Marchenko and D. Rolles, J. Chem. Phys. 149 (20), 204313 (2018).

[82] A. Guo, M. Burt and M. Brouard, Int. J. Mass Spectrom. 429, 121-126 (2018). 